\title{
Paternalismo em contraste com a recusa à transfusão de sangue: uma revisão integrativa
}

\author{
Paternalism in contrast to blood transfusion refusal: a integrative review
}

\author{
Paternalismo en contraste con el rechazo a la transfusión de sangre: una revisión \\ integradora
}

Bárbara Ferrarez Senra Costa e Silva ${ }^{1 *}$, Maria Luiza Werneck Elizeu ${ }^{1}$, Mariana Lima Rodrigues Pereira $^{1}$, Ully Jaques ${ }^{1}$, Douglas Almeida Costa ${ }^{1}$, Deborah Evelyn Miranda Medeiros Pedrosa ${ }^{1}$, Paulo Enrique Freitas Cruz², Vinícius Ferrarez Senra Costa e Silva², Márcia Farsura de Oliveira1.

\section{RESUMO}

Objetivo: Revisar as influências da Bioética Principialista (BP), da Constituição Federal de 1988 (CF88) e das Diretrizes Curriculares Nacionais para o Curso de Graduação em Medicina de 2014 (DCNs), nas características da relação médico-paciente, diante do cliente Testemunha de Jeová (TEJ), na recusa à hemotransfusão. Métodos: Foi feita uma revisão integrativa, através de uma busca de artigos publicados, nas bases de dados online Pubmed e SciELO, no período de 2010 a 2021. Resultados: A inclusão de 30 artigos, recrutados através do protocolo PRISMA, identificou que a BP influencia na garantia do direito das TEJ à liberdade religiosa e à saúde prevista na CF88. Além disso, observou-se o papel da DCNs, no auxílio à formação médica humanizada, na relevância do processo de trabalho em saúde, pautado pela bioética, e na relação médico-paciente, diante das discordâncias, quanto aos procedimentos em saúde. Considerações finais: Não há espaço para o paternalismo médico atualmente, sendo certo que, tão importante quanto tratar as mazelas corpóreas, também é o respeito aos limites impostos pelo sacrário da consciência individual do enfermo.

Palavras-chave: Educação médica, Bioética, Testemunhas de Jeová, Transfusão sanguínea.

\begin{abstract}
Objective: Review the influences of Principialist Bioethics (PB), the Federal Constitution of 1988 (CF88) and the National Curriculum Guidelines for the 2014 Medical Undergraduate Course (DCNs), on the characteristics of the doctor-patient relationship, in front of the client Witness of Jehovah (TEJ), in refusing blood transfusion. Methods: An integrative review was carried out, through a search for published articles, in the online databases Pubmed and SciELO, in the period 2010 to 2021. Results: The inclusion of 30 articles, recruited through the PRISMA protocol, identified that PB influences the guarantee of the TEJ's right to religious freedom and health provided for in CF88. In addition, the role of the DCNs was observed, in aiding humanized medical training, in the relevance of the health work process, guided by bioethics, and in the doctor-patient relationship, in the face of disagreements, regarding health procedures. Final considerations: There is currently no room for medical paternalism, and it is certain that, as important as treating bodily ailments, is also respect for the limits imposed by the sanctuary of the patient's individual conscience.
\end{abstract}

Keywords: Medical education, Bioethics, Jehovah's witnesses, Blood transfusion.

1 Faculdade Dinâmica do Vale do Piranga (FADIP), Ponte Nova - MG. *E-mail: barbaraferrarez@ hotmail.com

2 Centro Universitário UNIFAMINAS, Muriaé - MG. 


\section{RESUMEN}

Objetivo: Revisar las influencias de la Bioética Principialista (BP), la Constitución Federal de 1988 (CF88) y los Lineamientos Curriculares Nacionales para el Curso de Licenciatura en Medicina 2014 (DCNs), sobre las características de la relación médico-paciente, frente a la cliente Testigo de Jehová (TEJ), al rechazar la transfusión de sangre. Métodos: Se realizó una revisión integradora, mediante búsqueda de artículos publicados, en las bases de datos en línea Pubmed y SciELO, de 2010 a 2021. Resultados: La inclusión de 30 artículos, reclutados a través del protocolo PRISMA, identificó que BP influye en la garantía de el derecho del TEJ a la libertad religiosa y la salud previsto en el CF88. Además, se observó el papel de las DCNs, en la ayuda a la formación médica humanizada, en la relevancia del proceso de trabajo en salud, guiado por la bioética, y en la relación médico-paciente, ante los desacuerdos, en los procedimientos de salud. Consideraciones finales: Actualmente no hay lugar para el paternalismo médico, y es cierto que, tan importante como tratar las dolencias corporales, también es el respeto a los límites que impone el santuario de la conciencia individual del paciente.

Palabras clave: Educación médica, Bioética, Testigos de Jehová, Transfusión de sangre.

\section{INTRODUÇÃO}

As Testemunhas de Jeová (TEJ) iniciaram o movimento de estudos do Cristianismo, no século XIX, na Pensilvânia, nos Estados Unidos da América (EUA), onde anteriormente a terem reconhecimento de organização religiosa, consistiam em um pequeno grupo de pessoas que se reuniam para refletirem sobre a Bíblia. Escolheram o nome porquanto, segundo o seu livro sagrado: Jeová é o nome de Deus e testemunhas por se considerarem aqueles que afirmam a verdade sobre Jeová, o qual é apontado, por estes cristãos, como o Criador de todas as coisas (PETRINI C, 2014).

Quanto à doutrina, ocorre que, embora haja extrema coincidência entre a liturgia cristã tradicional e o conjunto dos elementos e práticas do rito das TEJ, existe discrepância substancial no que tange à não aceitação da hemotransfusão por parte destas últimas. Essa não aceitação se deve ao fato delas acreditarem que a abstenção do uso de sangue é algo importante e sagrado, sendo a única religião que possui essa doutrina (LIMA GL e BYK J, 2018; WEST JM, 2014).

Diante dessa peremptória recusa, o médico assistente se encontra diante do seu juramento hipocrático, o qual infere que ele deve cuidar do paciente de acordo com seu poder e entendimento dentro daquela área, sem causar prejuízo e sem induzir a morte por qualquer tipo de orientação, além de estar diante, de amplos e vigorosos conflitos da BP (beneficência, não maleficência, justiça e respeito à autonomia), os quais são de fácil incorporação no dia-a-dia médico, através do seu suporte, quase automático, para tomadas de decisão e, ainda, julgamentos morais. Esse embate está relacionado à autodeterminação do paciente, à dignidade da pessoa humana e ao direito à liberdade religiosa (TINGLE J, 2017; LIMA GL e BYK J, 2018).

Em contrapartida, está o paternalismo médico, que diz respeito à autoridade do profissional de medicina sobressair ante à autonomia do paciente. Ou seja, significa pensar um médico "pai", que atua com total autoridade. O paternalismo médico se constitui na forma de propor um tratamento que, objetivando beneficiar a pessoa, não leva em consideração as vontades e interesses alheios (JÚNIOR EAB, 2019).

Por anos a fio, a medicina foi caracterizada como a detentora das rédeas no tratamento, onde a vontade soberana do médico prevalecia. Valendo-nos do conceito do magistrado britânico e notável filósofo do direito Herbert Lionel Adolphus Hart, conhecido por seu trabalho, no estudo da moral e da filosofia política, o paternalismo seria a proteção dos enfermos de si mesmos, o que se tornou obsoleto, quando médicos alemães (principalmente entre 1930 e 1945) utilizaram técnicas experimentais em seres humanos, como, por exemplo, TEJ, valendo-se de teorias supremacistas (SIMÕES MA, 2016; CEZAR V, et al., 2017).

Os experimentos médicos chefiados por Josef Mengele, Eduard Wirths e Johann Kremer, culminaram na morte de milhões de pessoas. Tal conduta criminosa, imbuída de paternalismo e justificada como bom intento, encontrou no Tribunal Internacional de Nuremberg (1945) seu limite, o qual, além da benfazeja condenação 
dos referidos pesquisadores, atualmente, sustenta o estabelecimento de regras na experimentação científica em humanos e à bioética (RANGEL TLV, 2012). E, em 1948, a Declaração Universal dos Direitos Humanos orientou que já não havia mais espaços para a livre determinação médica, sendo imprescindível a construção de um plano terapêutico em conjunto com o doente, considerando, também, os seus preceitos religiosos (LEÃO AC, et al., 2019).

As Diretrizes Curriculares Nacionais para o Curso de Graduação em Medicina de 2014 (DCNs) preconizam a participação do cliente, no processo de escolha de terapêutica, além de defender, para o perfil do egresso que concluir o curso de graduação em Medicina, a formação pautada por humanismo, perfil generalista, desenvolvimento de habilidades e competências de características reflexivas e críticas, com subsequente atuação orientada por princípios éticos (CARNEIRO LA e PORTO CC, 2014).

Assim, o presente estudo intenciona fazer uma discussão, a partir de revisão integrativa da literatura científica, das influências da BP e das DCNs, nas características do processo de trabalho do profissional médico, considerando que antes da aprovação dessas últimas, houve a promulgação da Constituição Federal de 1988 (CF88), que deu ênfase à necessidade de o Estado garantir o direito à saúde e à liberdade religiosa para todos os cidadãos em território nacional.

\section{MÉTODOS}

O presente estudo trata-se de uma revisão integrativa de literatura, em que se realizou o levantamento de artigos a fim de sintetizar o conhecimento e a aplicabilidade do presente estudo. A CF88 e as DCNs foram recuperadas, respectivamente, no portal do Planalto do Governo Federal do Brasil e no endereço eletrônico do Ministério da Educação. Além disso, a pesquisa realizou uma busca de artigos publicados, nas bases de dados online United States National Library of Medicine (Pubmed) e Scientific Electronic Library Online (SciELO), no período de 2010 a 2021, com data limite até 7 de junho de 2021, devido à falta de publicações mais recentes que abordassem o tema proposto. Utilizou-se os descritores que se encontram representados na Tabela 1. Ademais, foram pesquisados artigos da área jurídica para complementar as bases deste trabalho.

Tabela 1 - Quantitativo de artigos obtidos em cada base de dados científica, de acordo com o descritor utilizado.

\begin{tabular}{|c|c|c|}
\hline Bases de dados & SciELO & Pubmed \\
\hline \multicolumn{3}{|l|}{ Descritor } \\
\hline "Jehovah's Witnesses" AND “Blood Transfusion" & 9 & 90 \\
\hline "Jehovah's Witnesses" AND "Bioethics" & 3 & 3 \\
\hline "Jehovah's Witnesses" AND “Education, Medical" & 0 & 1 \\
\hline "Testemunhas de Jeová" AND "Transfusão de Sangue" & 7 & 0 \\
\hline "Testemunhas de Jeová" AND “Bioética" & 3 & 0 \\
\hline "Testemunhas de Jeová" AND “Educação Médica" & 0 & 0 \\
\hline
\end{tabular}

Fonte: Silva BFSC, et al., 2021.

Desse modo, recorreu-se a quatro componentes: Educação Médica, Bioética, TEJ e Transfusão Sanguínea. Após a seleção, definiram-se os trabalhos que atendiam aos critérios de inclusão. Não se optou por restringir a linguagem na pesquisa de artigos. Estudos em duplicação foram excluídos posteriormente à comparação de: autoria, título, data e periódico de publicação. Todas as pesquisas significativas foram lidas e analisadas, as quais estão representadas no diagrama de pesquisa do grupo PRISMA (Principais Itens para Relatar Revisões Sistemáticas e Meta-análises) (Figura 1).

Os critérios de inclusão foram artigos originais, publicados nos últimos 10 anos (2010 - 2021), em qualquer idioma, e disponíveis em texto completo grátis. Em soma, foram considerados como critérios de exclusão: a) artigos duplicados; b) artigos que abordassem outro tema concomitante ao pesquisado e c) artigos que não 
abordavam o tema propriamente dito. Através dos resultados obtidos, foi realizada a leitura e seleção dos artigos através dos títulos e posteriormente leitura dos resumos, para, por fim, distinguir os textos que seriam lidos na íntegra. O período entre a identificação de trabalhos, nas bases científicas, e a compilação dos dados obtidos, correspondeu aos meses de março, abril e maio do ano 2021.

Os dados foram extraídos dos artigos e organizados da seguinte maneira: I) A BP; II) As TEJ, a Liberdade Religiosa e o Direito à Saúde Prevista na CF88; III) As DCNs; e VI) O Processo de Trabalho em Saúde, a Bioética e as Habilidades na Relação com Pacientes TEJ.

\section{RESULTADOS}

Foram recuperados 22 artigos, na base de dados SciELO, e 94 artigos, na base de dados PUBMED, com a utilização dos filtros. Com tal procedimento foram obtidos 116 artigos. Depois da aplicação dos critérios de inclusão e exclusão restaram 30 artigos, que foram incluídos neste estudo, conforme está descrito no fluxograma Prisma da Figura 1.

Figura 1 - Diagrama de fluxo dos resultados da pesquisa de literatura com base na declaração do PRISMA.

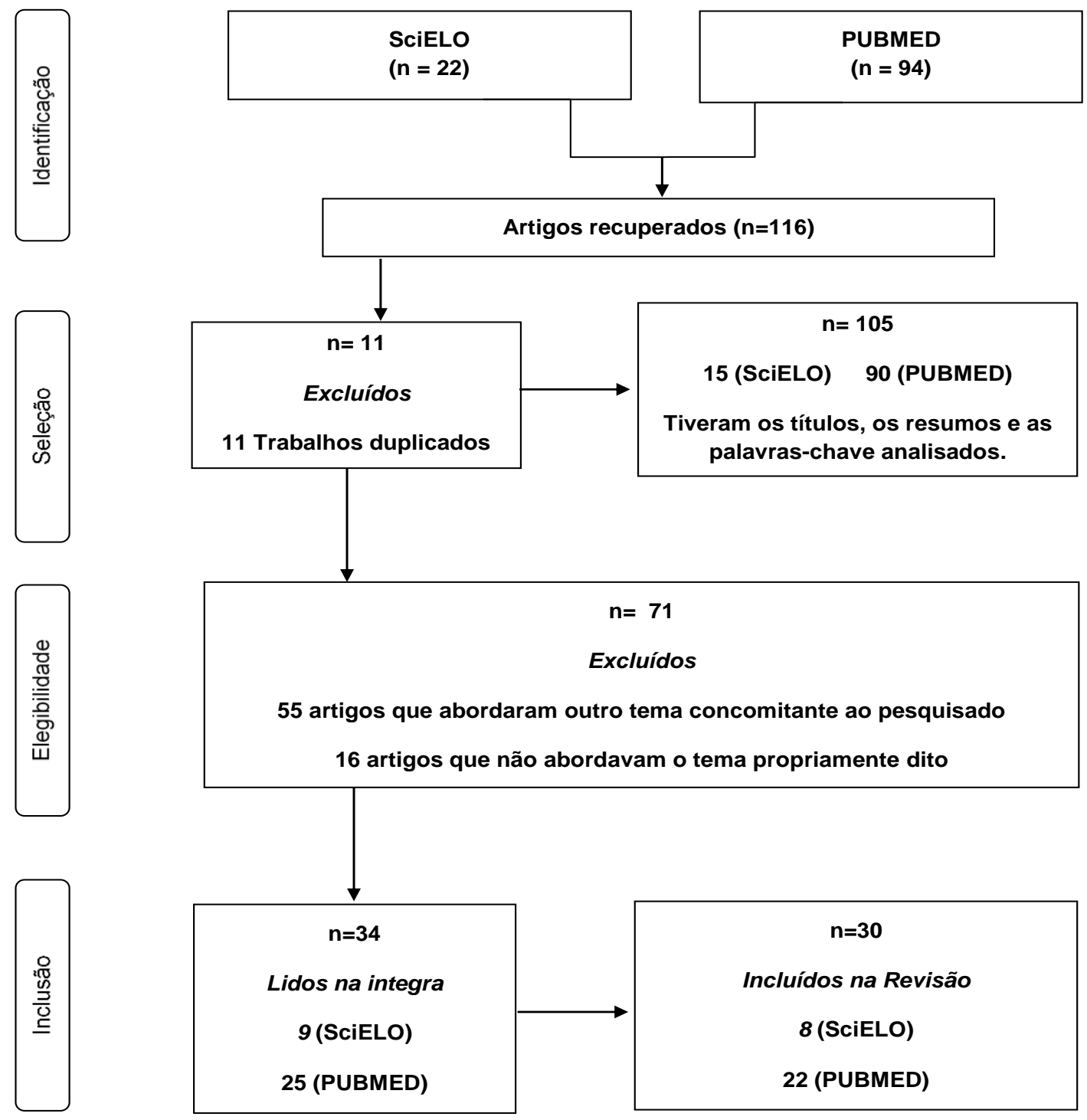

Fonte: Silva BFSC, et al., 2021.

Os dados obtidos pela revisão e o exame dos assuntos por meio de argumentos provenientes de fontes científicas se encontram descritos nos tópicos a seguir, e também expostas na Quadro 1. 
Quadro 1 - Síntese dos principais estudos selecionados para revisão.

\begin{tabular}{|c|c|c|c|}
\hline Autor (Ano) & Tipo de Estudo & $\begin{array}{c}\text { Base } \\
\text { Científica }\end{array}$ & Objetivo \\
\hline $\begin{array}{l}\text { AZAMBUJA LEO e } \\
\text { GARRAFA V, } 2010\end{array}$ & Estudo Original & Scielo & $\begin{array}{l}\text { Estudar o grau de conhecimento e aceitação de hemocomponentes e hemoderivados, pelas TEJ e propor } \\
\text { ferramentas bioéticas para o enfrentamento de eventuais conflitos éticos e morais nas relações com os } \\
\text { médicos. }\end{array}$ \\
\hline $\begin{array}{l}\text { BEVILACQUA G, et al., } \\
2020\end{array}$ & Estudo de Caso & PUBMED & $\begin{array}{l}\text { Analisar a Lei no } 219 \text { aprovada na Itália que fornece aos cidadãos instrumentos eficazes para expressar } \\
\text { suas decisões de saúde: as diretivas antecipadas de tratamento e os planos de cuidados partilhados. }\end{array}$ \\
\hline BLAKE V, 2012 & Estudo de Caso & PUBMED & $\begin{array}{c}\text { Analisar a recusa de menores em procedimentos que podem salvar suas vidas, levando em consideração } \\
\text { que os adolescentes não têm o direito de tomar suas decisões médicas. }\end{array}$ \\
\hline CARDEMIL HG, 2010 & Estudo Original & Scielo & Relatar normas ético-legais a respeito da autonomia e não discriminação dos pacientes. \\
\hline GARRAUD O, 2014 & Estudo de Revisão & PUBMED & $\begin{array}{c}\text { Avaliar se enfermarias de ginecologia e obstetrícia, estabelecem políticas para pacientes e parturientes } \\
\text { TEJ que recusam a transfusão de sangue. }\end{array}$ \\
\hline GOHEL M, et al., 2011 & Estudo de Revisão & PUBMED & $\begin{array}{l}\text { Revisitar e atualizar sobre os princípios da cirurgia "sem sangue" e as questões levantadas ao tratar } \\
\text { pacientes TEJ. }\end{array}$ \\
\hline GOUEZEC H, et al., 2016 & Estudo de Revisão & PUBMED & $\begin{array}{l}\text { Avaliar as modalidades de manejo dos médicos, frente à recusa da transfusão durante o atendimento a } \\
\text { uma TEJ }\end{array}$ \\
\hline $\begin{array}{l}\text { GRINBERG M e } \\
\text { ZLOTNIK GC, } 2011\end{array}$ & Estudo Original & Scielo & $\begin{array}{l}\text { Investigar se na prática clínica, há flexibilidade quando o médico quer transfundir sangue para salvar a vida } \\
\text { de um paciente que se recusa recebê-lo, evidenciando uma convivência inclusiva e corresponsável. }\end{array}$ \\
\hline HOFFMAN A, 2016 & Estudo de Revisão & PUBMED & $\begin{array}{c}\text { Ajudar os psicólogos a fornecerem consultas e apoio ético, investigando os valores da comunidade das } \\
\text { TEJ e a origem do tabu da transfusão de sangue. }\end{array}$ \\
\hline IRFAN A, et al., 2013 & Relato de Caso & PUBMED & $\begin{array}{l}\text { Abordar um caso sobre uma senhora TEJ, com história de anemia crônica, que sofre parada cardíaca e é } \\
\text { ressuscitada, a fim de mostras a necessidade de uma estrutura de atendimento e de revisar a história do } \\
\text { paciente, mesmo no âmbito de emergência. }\end{array}$ \\
\hline KUDELA M, et al., 2019 & Estudo Clínico & PUBMED & $\begin{array}{c}\text { Apontar os princípios da cirurgia conservadora de sangue e os aspectos médicos éticos, morais e legais } \\
\text { que envolvem as operações em TEJ. }\end{array}$ \\
\hline $\begin{array}{l}\text { LARA GF e PENDLOSKI } \\
\text { J, } 2013\end{array}$ & Estudo de Revisão & Scielo & Refletir sobre a prática dos enfermeiros diante do dilema ético: transfusão de sangue em TEJ. \\
\hline $\begin{array}{l}\text { LAWSON T e RALPH C, } \\
\qquad 2015\end{array}$ & Estudo de Revisão & PUBMED & $\begin{array}{c}\text { Examinar as questões perioperatórias e o manejo das TEJ e a descrição da sua história e suas crenças } \\
\text { junto com seu impacto sobre a ética e a lei, e as diferentes opções de tratamento durante o período } \\
\text { perioperatório. }\end{array}$ \\
\hline LIMA GL e BYK J, 2018 & Estudo de Revisão & Scielo & $\begin{array}{l}\text { Analisar as razões de conflito entre a atuação do médico e o direito de escolha e autonomia do paciente } \\
\text { TEJ e apresentar como o Judiciário, brasileiro e internacional, está decidindo sobre o tema. }\end{array}$ \\
\hline MICHELIS C, 2017 & Estudo Original & PUBMED & $\begin{array}{c}\text { Explorar os limites variáveis da acomodação multicultural e as condições que tornam possível a } \\
\text { compreensão, a colaboração e o compromisso dos TEJ perante seu reconhecimento atual, que pode } \\
\text { também ser alcançado por outros grupos de pacientes. }\end{array}$ \\
\hline
\end{tabular}




\begin{tabular}{|c|c|c|c|}
\hline Autor (Ano) & Tipo de Estudo & $\begin{array}{c}\text { Base } \\
\text { Científica }\end{array}$ & Objetivo \\
\hline MORENO JR, 2016 & Estudo de Revisão & PUBMED & $\begin{array}{l}\text { Analisar os conceitos éticos e jurídicos dentro do arcabouço jurídico vigente, e como as decisões tomadas } \\
\text { pelas pessoas, mesmo ainda com risco de vida, devem ser acatadas e protegidas por meio de remédio } \\
\text { judicial, sem prejuízo das exceções previstas, por lei. }\end{array}$ \\
\hline MUZNY P, 2011 & Estudo Original & PUBMED & $\begin{array}{c}\text { Examinar a legislação da Suíça, em que o direito do paciente informado e capaz à autodeterminação é } \\
\text { absoluto, e a diferente realidade na mentalidade de vários cuidadores, que consideram que a escolha do } \\
\text { paciente pode ser fatal, anulando sua vontade. }\end{array}$ \\
\hline PETRINI C, 2014 & Estudo de Revisão & PUBMED & Abordar os problemas associados à recusa de transfusões de sangue pelas TEJ. \\
\hline RAJTAR M, 2013 & Estudo Original & PUBMED & Documentar as experiências das TEJ em suas relações com o sistema de saúde alemão. \\
\hline RAJTAR M, 2018 & Estudo Etnográfico & PUBMED & $\begin{array}{l}\text { Mostrar como os estudos bioéticos atuais sobre autonomia relacional e a ética do cuidado podem ser } \\
\text { apropriados por antropólogos e sociólogos em seus estudos de fenômenos socioculturais e médicos. }\end{array}$ \\
\hline RIVERO RO, 2012 & Estudo de Revisão & PUBMED & $\begin{array}{l}\text { Oferecer uma análise ética e jurídica da autonomia moral e objeção de consciência do paciente TEJ e o } \\
\text { mesmo direito aos profissionais da saúde no manejo cirúrgico destes pacientes, com base na legislação } \\
\text { espanhola. }\end{array}$ \\
\hline SAGY I, et al., 2017 & Estudo Original & PUBMED & $\begin{array}{c}\text { Discutir detalhadamente sobre a tendencia atual de que a aversão das TEJ à transfusão poder ser } \\
\text { parcialmente justificada clinicamente. }\end{array}$ \\
\hline SANTANA ED, 2010 & Estudo Original & Scielo & $\begin{array}{l}\text { Avaliar em que medida o médico conhece, respeita, informa e aplica a Lei Geral de Saúde no que diz } \\
\text { respeito ao direito do paciente TEJ de recusar a transfusão (respeito à sua autonomia). }\end{array}$ \\
\hline $\begin{array}{l}\text { SCHARMAN CD, et al., } \\
\qquad 2017\end{array}$ & Estudo de Revisão & PUBMED & $\begin{array}{c}\text { Discutir várias áreas relevantes para o cuidado das TEJ, incluindo cuidados médicos "sem sangue" no } \\
\text { contexto de manejo perioperatório e intraoperatório, perda aguda de sangue, trauma, gravidez e } \\
\text { malignidade. }\end{array}$ \\
\hline $\begin{array}{l}\text { SILVA JUNIOR JM, et al., } \\
2012\end{array}$ & Estudo Original & Scielo & Investigar a epidemiologia e resultados em pacientes críticos sob o regime de transfusão restritiva. \\
\hline SYDOR DT, et al., 2013 & Estudo Original & PUBMED & $\begin{array}{c}\text { Investigar como a hierarquia na equipe da sala de cirurgia influencia a capacidade de um estagiário de } \\
\text { contestar uma decisão antiética de um anestesista experiente. }\end{array}$ \\
\hline $\begin{array}{l}\text { TAKASCHIMA AKK, et } \\
\text { al., } 2016\end{array}$ & Estudo Original & Scielo & $\begin{array}{l}\text { Estabelecer um protocolo de atendimento do paciente TEJ com ênfase no dever ético e legal do } \\
\text { anestesiologista. }\end{array}$ \\
\hline TINGLE J, 2017 & Estudo Original & PUBMED & $\begin{array}{l}\text { Discutir um importante caso judicial sobre a recusa de consentimento para uma transfusão de sangue por } \\
\text { uma TEJ e uma nova orientação sobre como cuidar de pacientes que recusam sangue. }\end{array}$ \\
\hline WEST JM, 2014 & Estudo de Revisão & PUBMED & $\begin{array}{c}\text { Revisar as crenças das TEJ em relação ao uso de sangue e hemoderivados e como garantir que esses } \\
\text { pacientes sejam tratados com ética. }\end{array}$ \\
\hline $\begin{array}{l}\text { WILLCOX TW, et al., } \\
2020\end{array}$ & Estudo Original & PUBMED & $\begin{array}{l}\text { Comparar o manejo intraoperatório dos fatores modificáveis de CEC e a incidência de LRA em pacientes } \\
\text { que recusaram transfusão de sangue em comparação com pacientes que aceitaram transfusão de sangue. }\end{array}$ \\
\hline
\end{tabular}

Legenda: CEC: circulação extracorpórea; LRA: Lesão renal aguda.

Fonte: Silva BFSC, et al., 2021 


\section{A Bioética Principialista}

A história da bioética, enquanto disciplina autônoma (sem se caracterizar como estudo da filosofia moral da área da saúde ou da ética da área da saúde), é demasiadamente recente, na qual o uso da expressão "Bioética", tal como denominamos hodiernamente, foi usado inicialmente no tratado "Bioethics: bridge to the future de Van Rensselaer Potter" (1971). Outro grande marco, para a bioética, foi o Relatório de Belmont, documento lavrado a partir de quatro anos de estudos de uma "Comissão Nacional para a Proteção de Sujeitos Humanos na Pesquisa Biomédica e Comportamental", instituída em 1974 que tinha como proposta fazer prevalecer três princípios éticos norteadores da conduta médico-paciente: princípio do respeito às pessoas; princípio da beneficência e princípio da justiça (DEJEANNE S, 2011).

O grande divisor de águas da bioética, porém, foi à publicação, em 1979, de "Princípios de ética biomédica" dos Autores: Beauchamp e Childress. Estes pensadores seguiam o entendimento fundado no Relatório Belmont anos antes, argumentando, basicamente, que conflitos morais podem ser mediados pela referência a algumas ferramentas morais, os princípios éticos. Nasce daí a expressão BP apresentando os fundamentos: justiça, autonomia, beneficência e não-maleficência. Destes quatro, dois se destacam como caríssimos ao recorte pretendido pelo presente trabalho: a autonomia e a beneficência (RIVERO RO, 2012).

A autonomia, elevada à condição de princípio da bioética, visa amparar o paciente de não ser vítima por duas vezes: de sua própria enfermidade e da proatividade médica malsã (indesejada e inoportuna). Quanto à beneficência, esta corresponde à indicação do médico, como um vetor único e inarredável, o qual age em busca de propiciar o bem ao enfermo (JÚNIOR EAB, 2019).

Em que pese a harmonia dos princípios esmiuçados, situações que envolvem a recusa de tratamento médico por ocasião da religiosidade, especialmente nos casos de hemotransfusão (geralmente casos de premente necessidade de preservação da vida), podem criar aparente conflito e a necessidade de ponderação de tais princípios para, à luz do caso concreto, fazer preponderar uma melhor deliberação (KUDELA M, et al., 2019)

Por outro lado, é importante destacar que os princípios não possuem abrangência irrestrita, sendo limitados. Assim, mesmo tendo ampla autonomia, o paciente não pode exigir a sua própria morte auxiliado por um médico (ainda que esteja em declínio crônico e sofrimento, o que se tem chamado de Eutanásia). $\mathrm{Na}$ mesma esteira, o médico não deve valer-se da beneficência para escolher, de forma divorciada do paciente, qual é o melhor tratamento para o usuário do serviço de saúde (o que se cunhou chamar de paternalismo médico), precisando respeitar os preceitos e a cultura da pessoa enferma. Tais assertivas ainda precisam ser mais estudadas e melhor abordadas, para que nem o paciente, e nem o médico sejam prejudicados diante de seus princípios (WEST JM, 2014).

\section{As TEJ, a Liberdade Religiosa e o Direito à Saúde Prevista na CF88}

Desde que surgiram (em 1870), as TEJ sofreram perseguição e preconceito. Exemplo disso é que foram vitimadas, em campos de concentração nazistas, e, após essa ocorrência, receberam o esquecimento de boa parte do mundo (diferentemente dos judeus, que foram e são reconhecidos como as grandes vítimas do holocausto nazista) (SIMÕES MA, 2016).

Além da perseguição nazista, perpetrada na segunda guerra mundial, os TEJ (conhecidos, ademais, como triângulos roxos) também sofrem perseguições difusas ao redor do mundo, tanto pela maneira como divulgam seu evangelho, quanto pelas idiossincrasias de sua cultura. A histórica perseguição sofrida por essa população tem um outro agravante: direitos completamente tolhidos, na escolha pela sujeição a procedimentos recomendados por médicos, que determinavam unilateralmente o tratamento ao qual deveria ser submetida, a despeito do que pensava (SIMÕES MA, 2016).

Após a Segunda Guerra Mundial, o processo civilizatório global foi impulsionado pela prevalência dos Direitos Humanos e, no Brasil, não foi diferente. A partir da redemocratização (1985/1988), o Brasil elevou a liberdade religiosa ao patamar de Direito Fundamental (não podendo ser suprimido da Constituição) (BRASIL, 1988). Como assegura o Art. 5ำ da CF88: 
"VI- é inviolável a liberdade de consciência e de crença, sendo assegurado o livre exercício dos cultos religiosos e garantida, na forma da lei, a proteção aos locais de culto e a suas liturgias;

VIII- ninguém será privado de direitos por motivo de crença religiosa ou de convicção filosófica ou política, salvo se as invocar para eximir-se de obrigação legal a todos imposta e recusar-se a cumprir prestação alternativa, fixada em lei" (BRASIL, 1988).

Os avanços civilizatórios são contundentes, levando-se em conta que, a Constituição Federal de 1967 (que precedeu A CF88) previa somente assegurar as crenças "que não contrariem a ordem pública e os bons costumes" (Art. 5 CFB 1967) (BRASIL,1967).

Dessa forma, o Brasil tornou-se um Estado Laico, tolerante a todas as religiões e credos, bem como um país que reconhece na religiosidade um traço marcante do exercício da cidadania, da liberdade e dos direitos individuais. Diante desse cenário, as TEJ possuem amplo respeito e amparo aos seus preceitos, assim como o direito à saúde, como previsto em lei, na qual se tornou um Direito Fundamental e Direito Social inerente a todo cidadão em território brasileiro, como pode ser observado no Art. 196:

"Art. 196. A saúde é direito de todos e dever do Estado, garantido mediante políticas sociais e econômicas que visem à redução do risco de doença e de outros agravos e ao acesso universal e igualitário às ações e serviços para sua promoção, proteção e recuperação" (BRASIL, 1967).

A CF88 também engendra e regulamenta o Sistema Único de Saúde (SUS), que garante acesso à saúde, irrestritamente, a todos os cidadãos, brasileiros ou estrangeiros, independentemente de ideologia, ainda que correspondam a pensamentos alternativos (não convencionais), sobre a prevalência de algum tratamento. Eis o caso das TEJ, que repelem veementemente a hemotransfusão, como procedimento terapêutico, o que requer do profissional de saúde proatividade e condução a métodos alternativos de tratamento ou, eventualmente, destinar o paciente a outro médico que queira e/ou possa atuar, como previsto no Código de Ética Médica, em concordância, ainda, com o que foi promulgado nas DCNs (SCHARMAN CD et al., 2017; MORENO JR, 2016).

\section{As DCNs}

As DCNs, na sua Seção I, expõe o seguinte aspecto- da Atenção à Saúde:

"Il - integralidade e humanização do cuidado por meio de prática médica contínua e integrada com as demais ações e instâncias de saúde, de modo a construir projetos terapêuticos compartilhados, estimulando o autocuidado e a autonomia das pessoas, famílias, grupos e comunidades e reconhecendo os usuários como protagonistas ativos de sua própria saúde" (BRASIL, 2014).

Considerando que a autonomia é a capacidade que o ser humano tem de optar pelas normas e valores que entende como válidos, sem intervenções heterônomas, o estudante de medicina deve estar ciente do seu futuro papel como médico, afim de sempre cuidar do paciente, informando-o sobre os recursos possíveis de tratamento para sua condição e os resultados prováveis, visando garantir que as informações dadas sejam compreendidas, principalmente aquelas referentes aos riscos e benefícios das intervenções e dos procedimentos realizados nas atividades em saúde, incentivando que os usuários dos serviços de saúde participem das decisões, à luz de suas próprias escolhas (GRINBERG M e ZLOTNIK GC, 2011). Cita-se, aqui, determinações das DCNs, que corroboram com a ideia descrita, quanto ao perfil de egresso médico:

"e) favorecimento da construção de vínculo, valorizando as preocupações, expectativas, crenças e os valores relacionados aos problemas relatados trazidos pela pessoa sob seus cuidados e responsáveis, possibilitando que ela analise sua própria situação de saúde e assim gerar autonomia no cuidado;

f) identificação dos motivos ou queixas, evitando julgamentos, considerando o contexto de vida e dos elementos biológicos, psicológicos, socioeconômicos e a 
investigação de práticas culturais de cura em saúde, de matriz afro-indígena-brasileira e de outras relacionadas ao processo saúde-doença" (BRASIL, 2014).

Portanto, o médico não deve estabelecer juízo de valor, mas ser empático, quanto à valorização e ao entendimento das crenças e dos valores trazidos pelos pacientes, respeitando a autonomia dos mesmos em suas decisões, como, também, está estabelecido na Declaração Universal sobre Bioética e Direitos Humanos, de 2005, em seu art. 50:

"Deve ser respeitada a autonomia dos indivíduos para tomar decisões, quando possam ser responsáveis por essas decisões e respeitem a autonomia dos demais. Devem ser tomadas medidas especiais para proteger direitos e interesses dos indivíduos não capazes de exercer autonomia" (UNESCO, 2006).

Desse modo, o desrespeito à vontade do paciente TEJ corresponde a uma violação à sua autodeterminação e, por isso, não deve estar presente na relação médico-paciente no processo de trabalho em saúde (RAJTAR M, 2018; WEST JM, 2014).

\section{O Processo de Trabalho em Saúde, a Bioética e as Habilidades na Relação com Pacientes TEJ}

Um profissional de saúde, em seu mister, deve ser guiado pelas premissas: o profissional não trata a doença, mas sim o doente. Ademais, cada paciente é um microcosmo, cada vida é um fim em si mesma e toda peculiaridade deve ser respeitada e o profissional milita em uma Ciência Humana, logo inexata, interpretativa e subjetiva. Assim, devido ao fato de que essas condições se unem uma simbiose interpretativa, culmina-se na ideia de que, para tratar do Ser Humano, é preciso humanidade. Nessa perspectiva, atributos como empatia, solidariedade, afetuosidade, compaixão e amor são tão importantes quanto técnica, conhecimento e experiência (GOUEZEC H, et al., 2016).

Em específico, a habilidade em abordar o paciente TEJ deve perpassar o cuidado dedicado pelo médico, em esclarecer que tudo o que puder ser feito ao paciente será realizado (com sua permissão), independentemente de aceitação à hemotransfusão ou não. Vale pontuar que a relação médico-paciente é bilateral e horizontal, quer dizer, não há hierarquia ou subordinação entre médico e paciente. De um lado, há a soberania da aceitação do tratamento proposto (por parte do paciente), por outro lado, tem-se a autonomia no exercício da profissão por parte do médico, que não pode ser compelido a orientar uma conduta, na qual não acredita (GOMES AMA, et al., 2012)

Os dados da literatura científica apontam o que concerne ao tratamento médico dedicado às TEJ que recusam a transfusão de sangue, por exemplo, alternativas que podem substituir ou, pelo menos, melhorar a ausência de transfusão. Estudos indicam que diversos líquidos se mostram eficazes expansores de volume do plasma: solução salina, dextrana, o Haemaccel, lactato de Ringer e hidroxietila de amido (HES; amidohidroxietil), além da eritoproietina (EPO) (LARA GF e PENDLOSKI J, 2013; WILLCOX TW, et al., 2020).

Caso, após uma explanação didática sobre essas informações, feita pelo profissional de saúde ao enfermo, a decisão do paciente cause conflitos de consciência ao médico, ele tem a autonomia de recusarse a manter o atendimento. Nesse caso, deve-se comunicar a decisão ao doente e observar a necessária continuidade do tratamento por outro médico (RAJTAR M, 2018).

O artigo 31 do Código de Ética Médica proíbe ao médico desrespeitar o direito do paciente (ou de seu representante legal) e decidir livremente sobre a execução de práticas diagnósticas ou terapêuticas, com exceção àqueles em situação de risco iminente de morte (JÚNIOR EAB, 2019).

Sendo que o pensamento oposto, em que o médico pode realizar o procedimento que bem entender é, hoje, completamente rechaçado inclusive, com sanção criminal em casos extremos, em que após a recusa do paciente em se submeter a algum tipo e tratamento/procedimento médico, o médico insistir, ele não estará violando os direitos constitucionais, as normas que regem a liberdade de crença, de religião e de consciência, além de estar mitigando o privilégio do direito à vida, não preservando o direito de escolha e autonomia dos indivíduos, cometendo um crime de constrangimento ilegal, previsto pelo artigo 146 do Código Penal (SANTANA ED, 2010; WEST JM, 2014; MORENO JR, 2016) 
Há, portanto, completa prevalência da autonomia do paciente em receber este ou aquele tratamento, salvo o risco iminente de morte do paciente, quando, somente assim, o médico possui liberdade plena para salvar a vida não importando o aceite de ninguém (GARRAUD O, 2014). Porém, a prevalência da autonomia do paciente não pode indicar uma obrigatoriedade médica a se sujeitar a realizar um tratamento no qual não acredita. Em casos de conflitos entre as partes, o médico deve se abster de dar continuidade ao tratamento, assegurando-se de que o paciente seja assistido por outro profissional (RAJTAR M, 2018).

\section{CONSIDERAÇÕES FINAIS}

A presente pesquisa identificou que inexistem espaços para o paternalismo médico atualmente, sendo certo que, tão importante quanto tratar as mazelas corpóreas, também é o fato de respeitar os limites impostos pelo sacrário da consciência individual de cada paciente, conforme orientações da BP, da CF88 e das DCNs. Diante das TEJ, o médico deve recusar o tratamento com hemotransfusão ou priorizar tratamentos alternativos à mesma, desde que haja um substituto efetivo, pois, violar a decisão dos pacientes, além de inaceitável, pode caracterizar crime, além de falta grave sancionada junto aos órgãos oficiais. Vale ressaltar, que o médico deve acatar a decisão do enfermo, sem se deixar levar pelo paternalismo.

\section{REFERÊNCIAS}

1. AZAMBUjA LEO, GARRAFA V. Testemunhas de Jeová ante o uso de hemocomponentes e hemoderivados. Rev. Assoc. Med. Bras., 2010; 56(6):705-9.

2. BEVILACQUA G, et al. Shared care plan: an extraordinary tool for the personalization of medicine and respect for selfdetermination. Acta Biomed., 2020; 92(1):e2021001.

3. BLAKE V. Minors' Refusal of Life-Saving Therapies. AMA J. Ethics. 14(10):792-6.

4. BRASIL. Constituição (1967). Congr. Câmara dos Deputados. Secr. da Presidência. Brasil; 1967. 706p. Disponível em: http://www.planalto.gov.br/ccivil_03/constituicao/constituicao67.htm. Acessado em: 28 de julho de 2021.

5. BRASIL. Constituição (1988). Constituição da República Fed. do Bras. Brasília: Senado Federal, Coordenação de Edições Técnicas, 1988; p. 496. Disponível em: http://www.planalto.gov.br/ccivil_03/constituicao/constituicao.htm. Acessado em: 28 de julho de 2021.

6. BRASIL. Resolução CNE/CES 3/2014. Diário Of. da União. Brasilia: 23 de junho de 2014 - Seção 1, $2014 ; 11 p$. Disponível em: 14\&ltemid=3019. Acessado em: 28 de julho de 2021.

7. CARDEMIL HG. Consideraciones Éticas en El Tratamiento Médico a Testigos de Jehová. Acta Bioeth., 2010; 16(1).

8. CARNEIRO LA, PORTO CC. Saúde mental nos cursos de graduação: interfaces com as diretrizes curriculares nacionais e com a reforma psiquiátrica. Cad. Bras. Saúde Ment. Florianópolis, 2014; 6(14): 150-67.

9. CEZAR V, et al. Breves notas sobre o que Hart ainda tem a nos oferecer: o pensamento jurídico hartiano entre a crítica da moralidade e a construção de um novo positivismo. Jure, 2017; 16(28):177-94.

10. DEJEANNE S. Os fundamentos da bioética e a teoria principialista. Thaum. Rev. Online Filos, 2011; 4(7):32-45.

11. GARRAUD O. Jehovah's Witnesses and blood transfusion refusal: what next? Blood Transfus. SIMTI Servizi, 2014; 12(1): 402-3.

12. GOHEL M, et al. Avoiding blood transfusion in surgical patients (including Jehovah's Witnesses). Ann. R. Coll. Surg. Engl., 2010; 93(6): 429-31.

13. GOMES AMA, et al. Relação médico-paciente: Entre o desejável e o possível na atenção primária à saúde. Physis. IMS-UERJ, 2012; 22(3):1101-19.

14. GOUEZEC H, et al. Physicians' perception on taking care of Jehovah's witnesses. Transfus. Clin. Biol., 2016; 23(4):196-201.

15. GRINBERG M, ZLOTNIK GC. Posturas da Relação Médico-paciente Testemunha de Jeová. Arq. Bras. Cardiol., 2011; 97(3):e56-7.

16. HOFFMAN A. Jehovah's Witness parents' refusal of blood transfusions: Ethical considerations for psychologists. J. Health Psychol., 2016; 21(8):1556-65.

17. IRFAN A, et al. The value of history and goals of care with code status; even in an emergency setting. Case Reports., 2013; 1 :bcr2012006962.

18. JÚNIOR EAB. Código de ética médica: comentado e interpretado. Timburi, SP: Editora Cia do eBook, 2019, 835p.

19. KUDELA M, et al. Medical ethical moral and legal aspects of Jehovah's Witnesses operations. Ces. Gynekol, 2019; 84(1):23-7.

20. LARA GF, PENDLOSKI J. Os Enfermeiros diante do dilema ético: Transfusão de sangue em Testemunhas de Jeová. Rev. UNINGÀ, 2013; 16(1): 70-7. 
21. LAWSON T, RALPH C. Perioperative Jehovah's Witnesses: a review. Br. J. Anaesth., 2015; 115(5):676-87.

22. LIMA GL, BYK J. Trauma e transfusão sanguínea precoce: o desafiante manejo de hemorragias em Testemunhas de Jeová. Rev. Col. Bras. Cir., 2018; 45(6).

23. MICHELIS C. Transfusion Refusal and the Shifting Limits of Multicultural Accommodation. Qual. Health Res., 2017; 27(14): 2150-61.

24. MORENO JR. Legal Considerations for Nurses. The Principle of Autonomy and The Rejection of Therapy with Blood: Jehovah's Witnesses. Rev. Enferm., 2016; 39(2):30-6.

25. MUZNY P. Respecting the Will of the Patient: Between Illusions and Realities: Table 1. Pain Med., 2011; 12(11):16848.

26. ORGANIZAÇÃO DAS NAÇÕES UNIDAS (UNESCO). Declaração Universal sobre Bioética e Direitos Humanos. Tradução: Ana Tapajós e Mauro Machado do Prado. 2006. Disponível em: https://bvsms.saude.gov.br/bvs/publicacoes/declaracao_univ_bioetica_dir_hum.pdf. Acessado em: 28 de julho de 2021.

27. PETRINI C. Ethical and legal aspects of refusal of blood transfusions by Jehovah's Witnesses, with particular reference to Italy. Blood Transfus., 2014; 12(1): s395-s401.

28. RAJTAR M. Bioethics and religious bodies: Refusal of blood transfusions in Germany. Soc. Sci. Med., 2013; 98: 2717.

29. RAJTAR M. Relational autonomy, care, and Jehovah's Witnesses in Germany. Bioethics, 2018; 32(3):184-92.

30. RIVERO RO. Moral autonomy and conscientious objection in the surgical management of Jehova's witnesses. Cuad. Bioet., 2012; 23(79): 657-73.

31. SAGY I, et al. Reflections on Cultural Preferences and Internal Medicine: The Case of Jehovah's Witnesses and the Changing Thresholds for Blood Transfusions. J. Relig. Health. Springer US, 2017; 56(2):732-8.

32. SANTANA ED. Conocimiento de la ley general de salud respecto de las transfusiones sanguíneas en médicos y pacientes testigos de jehová del hospital Dr. Darío Contreras de República Dominicana. Acta Bioeth., 2010;16(1).

33. SCHARMAN CD, et al. Treatment of individuals who cannot receive blood products for religious or other reasons. Am. J. Hematol., 2017; 92(12):1370-81.

34. SILVA JUNIOR JM, et al. Red blood cell transfusions worsen the outcomes even in critically ill patients undergoing a restrictive transfusion strategy. Sao Paulo Med. J., 2012; 130(2):77-83.

35. SIMÕES MA. As Testemunhas de Jeová e o Regime Nazista: uma análise das causas ideológicas do conflito. Dissertação (Monografia em Ciências Sociais) - Faculdade de Ciências Socuais. Pontifícia Universidade Católica de São Paulo, São Paulo, 2016; 155p.

36. SYDOR DT, et al. Challenging authority during a life-threatening crisis: the effect of operating theatre hierarchy. Br. J. Anaesth., 2013; 110(3): 463-71.

37. TAKASCHIMA AKK, et al. Ethical and legal duty of anesthesiologists regarding Jehovah's Witness patient: care protocol. Brazilian J. Anesthesiol (English Ed.), 2016; 66(6): 637-41.

38. TINGLE J. Patient consent and conscientious objection. Br. J. Nurs., 2017; 26(2):118-9.

39. WEST JM. Ethical issues in the care of Jehovah's Witnesses. Curr. Opin. Anaesthesiol, 2014; 27(2): $170-6$.

40. WILLCOX TW, et al. Cardiopulmonary bypass management and acute kidney injury in 118 Jehovah's Witness patients: a retrospective propensity-matched multicentre cohort from 30,942 patients. Perfus. (United Kingdom). SAGE Publications Ltd., 2020; 35(8): 833-41 